\title{
白马雪山阳坡林线方枝柏种群的生态特征
}

\author{
张桥英 ${ }^{12} \quad$ 张运春 $^{1}$ 罗 鹏 $^{1}$ 王 乾 $^{1}$ 吴 $\quad$ 宁 $^{1}{ }^{*}$ \\ （1 中国科学院成都生物研究所, 成都 610041) (2 中山大学有害生物控制与利用国家重点实验室, 广州 510275)
}

摘 要 方枝柏 (Sabina saltuaria) 是青藏高原特有林线树种。对白马雪山阳坡海拔 $4390 \mathrm{~m}$ 以上林线 $0.42 \mathrm{hm}^{2}$ 样地 方枝柏种群进行每木调查, 分析了种群结构、数量动态及空间分布格局。结果表明:1)在种群生物学特征、环境因 子及人为干扰等综合影响下 林线区方枝柏幼苗数量非常少。幼树在种群中占了很大比重, 种群个体数随径级的 增加而逐渐减少, 密度为成年树 > 幼树 > 幼苗 ,2) 种群存活曲线介于 Deevey- II 型和 Deevey- III 型之间, 种群结构更 接近稳定型。死亡率曲线和消失率曲线都在龄级 III 出现一个高峰, 可能是由于方枝柏处于青壮年期, 个体对营养 空间的需求不断增大 对空间、光照和养分等生存因子的激烈竞争引起的自疏过程, 导致死亡率有所上升 ;3) 方枝 柏种群各龄级的空间格局基本上是聚集型, 随龄级增加, 聚集尺度和强度都增加; 各龄级关系密切, 都呈显著正相 关, 其中幼苗和成年树的相关性最强 幼苗和幼树相关性最弱。林线方枝柏种群空间分布格局是种群生物学特性、 环境条件及人为干扰等因素共同作用的结果, 这种格局有利于整个种群的生存和发展, 也是高山生态系统恶劣生 境中种群的一种适应对策。该研究采用森林罗盘仪与测距仪相结合进行每木定位的方法, 准确度高且大大降低工 作强度, 是高海拔区恶劣条件下开展类似工作的推荐之法。研究结果也再次体现了空间点格局分析方法在格局研 究中的有效性和优越性。

关键词 种群结构 特定时间生命表 生存分析 空间点格局 高山林线

\section{ECOLOGICAL CHARACTERISTICS OF A SABINA SALTUARIA POPULATION AT TIMBERLINE ON THE SOUTH-FACING SLOPE OF BAIMA SNOW MOUNTAIN , SOUTHWEST CHINA}

\author{
ZHANG Qiao-Ying ${ }^{1}{ }^{2}$, ZHANG Yun-Chun $^{1}$, LUO Peng ${ }^{1}$, WANG Qian ${ }^{1}$, and WU Ning ${ }^{1}{ }^{*}$ \\ ${ }^{I}$ Chengdu Institute of Biology , Chinese Academy of Sciences , Chengdu 610041 , China , and ${ }^{2}$ State Key Laboratory of Biocontrol , Sun Yat-Sen Uni- \\ versity, Guangzhou 510275 , China
}

\begin{abstract}
Aims Alpine timberlines are ecotones highly sensitive to disturbances and environmental changes that have become a major focus of global climate change research. Sabina saltuaria is an endemic timberline tree in the Qinghai-Tibetan Plateau. It occurs mainly on south-facing slopes and may be useful in ecological restoration of the high-altitude ecosystem. Understanding its ecological characteristics is necessary for understanding its adaptation strategies, as well as the causes and dynamics of timberline patterns in the region.

Methods We established one $0.42 \mathrm{hm}^{2}$ sample plot at timberline $>4390 \mathrm{~m}$ on the south-facing slope of Baima Snow Mountain, Northwest Yunnan Province. We recorded height, DBH (diameter at breast height) or diameter at base for seedlings and saplings, and location of each individual. We used a compass and electronic distance-measuring tool to record the location of each individual. We analyzed the size and $D B H$ structure , time-specific life table , survivorship rate, mortality and hazard rate, spatial point pattern of individuals in each age-class and the co-relationships between different age classes.

Important findings Few seedlings occur at timberline in the area of our field survey (about $17 \mathrm{ind} \mathrm{hm}^{-2}$, only $1 / 6$ and $1 / 14$ of that of saplings and trees, respectively). Densities declined from trees to saplings to seedlings , and the number of individuals decreased gradually with greater $D B H$. The survivorship curve was between Deevy type II and III. The mortality rate curve was similar to the hazard rate curve ; both exhibited a peak of mortality at $6-9 \mathrm{~cm} D B H$ ). Spatial point pattern analysis indicated that most individuals of different age classes had a clumped distribution at different scales (the older the trees, the more aggregative the distri-
\end{abstract}

收稿日期 : 2006-04-18 接受日期 : 2006-07-16

基金项目 : 本研究由中国科学院重大项目 (KSCX1-07)、重要方向项目 (KSCX2-01-09)、国家 十五”攻关项目 (2001BA606A-05) 和四川省青年 科技基金项目 (03ZQ026-043)

美国大自然保护协会德钦办公室为野外工作提供大量便利, 中国科学院南京土壤研究所江西红壤站完成部分土样分析, 谨致谢忱

* 通讯作者 Author for correspondence E-mail : wuning@cib.ac.cn

E-mail of the first author : zhangqy@ cib.ac.cn 
bution pattern) ; however, seedlings had a random distribution at the scale of $<14 \mathrm{~m}$. Also the relationships among individuals in different age classes were all significantly correlated with each other. The associations were more significant between seedlings and trees.

Key words population structure, time-specific life table , survival analysis , spatial point pattern , alpine timberline

种群生态学是研究现代生态学各层次间的联结 和枢纽 (江洪, 1992)。种群动态是种群生态学的核 心 种群数量变动和空间分布规律是种群生态学的 主要研究任务。生命表和存活曲线是进行种群统计 和研究种群数量动态变化的重要工具, 生命表结构 分析是解释种群变化的前提, 而生命表结构又是这 一分析的首要工作 (间桂琴等, 2001; 徐学红等, 2005)。种群空间格局分析是研究种群特征、种群间 相互作用以及种群与环境关系的重要手段，不同的 格局类型可以反映种群利用环境资源的状况、揭示 种群生殖生物学内涵, 在生态学中一直是研究的热 点之一（张金屯,1998; Dale,1999 ; Condit et al. , 2000)。

高山林线 (Alpine timberline) 是一类典型的生态 交错带，代表陆地上最明显的受非生物因素控制的 过渡带, 因其特殊的结构和功能而对全球气候变化 具有高度敏感性, 成为高山生态学研究的热点之一。

(Tranquillini ,1979 ;Lloyd \& Graumlich ,1997 ;Körner , 1998 ,1999)。青藏高原分布着世界上海拔最高的高 山林线, 但对这一区域林线种群生态学的研究相对 较缺乏，迄今仅有少量报道( 沈泽昊等, 2001 ;陈文年 等 ,2003 程伟等 ,2005a 2005b)。

方枝柏 (Sabina saltuaria) 是我国特有树种, 主要 分布于川西、滇西北和西藏等地区高山或高原丘状 山岭的阳坡或半阳坡(中国植被编辑委员会, 1980), 具有喜光、耐寒、耐旱、抗风等特性 (四川植被协作 组, 1980），对于高山生态系统的生态恢复具有重要 意义。由于自然和人类活动的影响, 目前尚存的方 枝柏原始林已较为零星, 多呈块状分布, 云南仅在德 钦县周围高山有成片森林, 是该区阳坡森林群落的 建群种, 在林线以单种群存在 (吴宁和刘照光, 1998)。对方枝柏的相关研究仅停留在《中国植被》
(中国植被编辑委员会, 1980) 、《四川植被》(四川植 被协作组，1980) 等文献的一般描述方面, 尚未见种 群生态学方面的研究报道。本研究以白马雪山阳坡 林线方枝柏种群为研究对象, 从种群生态学角度对 种群结构、种群动态、空间分布等方面进行系统的分 析研究，以期了解林线区方枝柏种群的生态特征及 其对环境的适应, 为深入探讨青藏高原东缘高山林 线格局和动态变化奠定基础。

\section{1 研究方法}

\section{1 样地概况}

研究地白马雪山国家级自然保护区地处青藏高 原南延部分横断山脉中部, 云南省西北部迪庆藏族 自治州德钦和维西县境内, 地势雄伟陡峭, 以极高 山、高山为主,地理坐标为 $27^{\circ} 24^{\prime} \sim 28^{\circ} 36^{\prime} \mathrm{N}$, $98^{\circ} 57^{\prime} \sim 99^{\circ} 25^{\prime} \mathrm{E}$, 总面积为 $281640 \mathrm{hm}^{2}$ 。主峰扎拉 雀尼海拔为 $5429 \mathrm{~m}$, 平均海拔为 $4000 \mathrm{~m}$ 以上, 最低 海拔为金沙江边 $2040 \mathrm{~m}$, 相对高差为 $3389 \mathrm{~m}$ 。处 于低纬度高海拔地带, 为寒温带山地季风气候“, 立 体” 和季风两大气候特征显著, 干湿季明显, 雨量少 而集中，气候随海拔的升高而变化，形成河谷温暖干 燥、山地严寒的特点, 自然景观垂直带谱十分明显。 据德钦 (海拔 $3485 \mathrm{~m}$ ) 气象数据: 年平均气温为 4.7 ${ }^{\circ} \mathrm{C}, 7$ 月均温为 $11.7{ }^{\circ} \mathrm{C} ; 1$ 月均温为 $-3.1{ }^{\circ} \mathrm{C}$ (据此, 研究地年均温约为 $-1.3{ }^{\circ} \mathrm{C}, 7$ 月均温约为 $5.7{ }^{\circ} \mathrm{C}, 1$ 月均温为 $\left.-9.1{ }^{\circ} \mathrm{C}\right)$ 。降雪天数达 $51 \mathrm{~d}$ 积雪天数达 $50 \mathrm{~d}$ 积雪厚 $52 \mathrm{~cm}$ 。年平均降水量为 $650.5 \mathrm{~mm}$, 无 霜期为 $129 \mathrm{~d}$ (云南省林业厅,2003)。样地基本特征 见表 1 。

群落结构简单，乔木层仅由方枝柏形成，最大高 度 $6 \mathrm{~m}$,多数低于 $4 \mathrm{~m}$, 郁闭度很低, 在 0.2 左右; 灌 木层主要是高山柏 ( Sabina squamata), 样地中仅 5

表 1 白马雪山阳坡林线方枝柏种群研究地点的特征

Table 1 The habitat characteristics of Sabina saltuaria at timberline of south-facing slope in the Baima Snow Mountain

\begin{tabular}{|c|c|c|c|c|c|c|c|}
\hline $\begin{array}{c}\text { 海拔 } \\
\text { Altitude }(\mathrm{m})\end{array}$ & $\begin{array}{c}\text { 样地大小 } \\
\text { Plot size }\left(\mathrm{m}^{2}\right)\end{array}$ & $\begin{array}{c}\text { 郁闭度 } \\
\text { Canopy coverage }\end{array}$ & $\begin{array}{l}\text { 坡向 } \\
\text { Aspect }\end{array}$ & $\begin{array}{l}\text { 坡度 } \\
\text { Slope }\end{array}$ & $\begin{array}{l}\text { 土壤类型 } \\
\text { Soil type }\end{array}$ & $\begin{array}{c}\text { 土壤 } \mathrm{pH} \text { 值 } \\
\text { Soil } \mathrm{pH}\end{array}$ & $\begin{array}{c}\text { 土壤有机质 } \\
\text { Organic mass in soil }\left(\mathrm{g} \mathrm{kg}^{-1}\right)\end{array}$ \\
\hline 4394 & 4200 & 0.2 & 235 & 28 & $\begin{array}{c}\text { 山地棕壤 } \\
\text { Mountain brown soil }\end{array}$ & $4.97 \sim 6.24$ & $74.2 \sim 86.3$ \\
\hline
\end{tabular}


丛, 高度 $1 \mathrm{~m}$ 左右; 草本层包括藏西大戟 (Euphorbia stracheyi)、黄总花草 ( Spenceria ramalana)、鹅线萎陵 菜 (Potentilla anserina) 、钉柱委陵菜 ( $P$. saundersiana)、圆叶堇菜 (Viola pseudo-bambusetorum)、苔草 (Carex spp.) 、嵩草 (Kobresia spp.) 等, 高度多在 20 $\mathrm{cm}$ 以下, 盖度小于 $20 \%$ 。

\section{2 野外调查}

野外工作于 2005 年 6 月中下旬进行。研究地 设置在白马雪山阳坡林线区, 以海拔 $4394 \mathrm{~m}$, 以 $28^{\circ} 21^{\prime} 55^{\prime \prime} \mathrm{N} 、 99^{\circ} 02^{\prime} 04^{\prime \prime} \mathrm{E}$ 为中心设置样方, 在野外调 查中根据样地的实际情况, 以垂直于坡向的方向和 沿着坡向的方向设置样方面积为 $70 \mathrm{~m} \times 60 \mathrm{~m}$, 通过 森林罗盘仪测量树木基部与中心点的方位角和高度 角、测距仪测量树木距离中心点的距离来进行每木 调查。调查内容包括树高、胸径、南北向的冠幅, 在 大样方中随机设置 4 个 $5 \mathrm{~m} \times 5 \mathrm{~m}$ 的小样方, 调查灌 木层物种组成、高度和盖度, 并在其中设置 $1 \mathrm{~m} \times 1$ $\mathrm{m}$ 小样方测定草本层高度和盖度。在样地的不同方 位选取小样地, 清除掉表层的覆盖物后, 在每块小样 地内 $0 \sim 15 \mathrm{~cm}$ 的深度挖取一个 $5 \mathrm{~cm} \times 5 \mathrm{~cm} \times 5 \mathrm{~cm}$ 的土样 共取 5 个土样带回实验室分析。

\section{3 种群径级的确定}

由于方枝柏木材坚硬, 加上林线区植株矮小且 侧枝较多, 不易钻取木芯, 因而要确定个体年龄较为 困难，而通过伐木来得到每个个体年龄又破坏性太 大, 鉴于此, 本研究采用径级结构代替年龄结构分析 种群结构。方枝柏种群的径级结构直接以胸径大小 为标准, $0 \sim 3 \mathrm{~cm}$ 为第一径级, 胸径每增加 $3 \mathrm{~cm}$ 径级 增加 1 级, 并绘制种群径级结构图。

\section{4 生命表编制及曲线绘制}

由于方枝柏生长周期长，不可能追踪所有的个 体生长周期，因而选择通过现实不同年龄阶段的个 体数量来推测种群时间上的动态过程。但由于测定 每一种群个体年龄较困难, 参考已有研究方法(问桂 琴等 ,2001 徐学红等, 2005)，以径级代替龄级, 虽然 龄级和径级有所不同, 但在同一环境下同一树种的 龄级和径级对环境的反应规律具有一致性 (Frost \& Rydin ,2000)。把树木径级从小到大的顺序看作是 时间顺序关系，第一径级对应第 I 龄级，第二径级对 应第 II 龄级, 以此类推, 统计各龄级株数, 并将数据 标准化 编制白马雪山林线方枝柏种群特定时间生 命表 进而分析其动态变化。以方枝柏的特定时间 生命表为基础, 以标准化存活数 $l_{x}$ 为纵坐标, 以径 级相对的龄级为横坐标绘制存活曲线, 以 $q_{x} 、 K_{x}$ 为
纵坐标，以径级相对的龄级为横坐标绘制死亡率曲 线和消失率曲线。

\section{5 格局分析}

根据如下计算方法将方位角、高度角和距离转 换为每棵方枝柏的坐标。

$$
\begin{aligned}
& \left\{\begin{array}{l}
x=L \cos \theta \sin \phi \\
y=L \cos \theta \cos \phi \\
z=L \sin \theta+h
\end{array}\right. \\
& \left\{\begin{array}{l}
x^{\prime}=-x \cos A-y \sin A \cos \mu+z \sin A \sin \mu \\
y^{\prime}=x \sin A-y \cos A \cos \mu+z \cos A \sin \mu \\
z^{\prime}=y \sin \mu+z \cos \mu
\end{array}\right.
\end{aligned}
$$

其中, $L$ 为罗盘仪到树木基部的距离, $\theta$ 为树木 基部相对于罗盘仪的高度角, $\phi$ 为树木基部的方位 角, $h$ 为罗盘仪距地面的垂直高度, $\mu$ 为坡度, $A$ 为 坡向。

由于林线区地形相对复杂,需要不断移动罗盘 仪的位置来给样方内所有树木定位, 须将计算出的 初始坐标通过以下转换从而将不同坐标系下测量的 结果统一到一个坐标系中, 即 : $X=x_{i}{ }^{\prime}-\sum_{i=1}^{i} t_{i}, Y=$ $y_{i}{ }^{\prime}-\sum_{i=1}^{i} t_{i}$ 将算出的 $(X, Y)$ 作为样方中方枝柏个体 的坐标。

在判断方枝柏种群不同龄级分布格局时, 结合 种群生长状况且为了便于分析将其分成 3 级, 胸 径 $\leqslant 2.5 \mathrm{~cm}$ 的作为幼龄个体, 将其中高度 $\leqslant 30 \mathrm{~cm}$ 的方枝柏划为幼苗, 高度 $>30 \mathrm{~cm}$ 的划为幼树, 胸径 $>2.5 \mathrm{~cm}$ 的划为成年树。格局分析采用在种群空 间分布格局分析方面备受推崇的空间点格局分析法 (SPPA 亦称 Ripley's $K$ 系数法) (Getis \& Franklin， 1987 张金屯和孟东平，2004 赵常明等 2004)。

\section{2 研究结果与分析}

\section{1 方枝柏种群径级分布}

方枝柏种群径级分布见图 1。在白马雪山阳坡 林线区 整个种群的径级分布呈直线下降趋势, $0 \sim 3$ $\mathrm{cm}$ 以及 $3 \sim 6 \mathrm{~cm}$ 径级的方枝柏分别占了 $1 / 3$ 和 $1 / 4$, 个体数随径级的增加而逐渐减少, 胸径 $>18 \mathrm{~cm}$ 的 方枝柏个体仅有 2 个。

\section{2 方枝柏种群特定时间生命表}

2.3 存活曲线, 死亡率曲线及消失率曲线

方枝柏种群存活曲线、死亡率和消失率曲线见 图 2 和图 3。根据 Deevey 的划分, 存活曲线可以分 为 3 种类型 (江洪 ,1992) :Deevey- I 型、 Deevey- II 型、 Deevey- III 型曲线。从图2来看, 白马雪山阳坡林线 
表 2 白马雪山阳坡林线方枝柏种群特定时间生命表

Table 2 The time-specific life table of Sabina saltuaria at timberline of south-facing slope in Baima Snow Mountain

\begin{tabular}{|c|c|c|c|c|c|c|c|c|c|c|c|c|c|c|}
\hline 龄级 & Age class & 径级 & $D B H$ class $(\mathrm{cm})$ & $x$ & $\Delta_{x}$ & $a_{x}$ & $l_{x}$ & $d_{x}$ & $q_{x}$ & $L_{x}$ & $T_{x}$ & $e_{x}$ & $S_{x}$ & $K_{x}$ \\
\hline & I & & $0 \sim 3$ & 1.5 & 3 & 48 & 1000.00 & 229.17 & 0.23 & 885.42 & 2500.00 & 2.50 & 0.77 & 0.26 \\
\hline & II & & $3 \sim 6$ & 4.5 & 3 & 37 & 770.83 & 187.50 & 0.24 & 677.08 & 1614.58 & 2.09 & 0.76 & 0.28 \\
\hline & III & & $6 \sim 9$ & 7.5 & 3 & 28 & 583.33 & 270.83 & 0.46 & 447.92 & 937.50 & 1.61 & 0.54 & 0.62 \\
\hline & IV & & $9 \sim 12$ & 10.5 & 3 & 15 & 312.50 & 104.17 & 0.33 & 260.42 & 489.58 & 1.57 & 0.67 & 0.41 \\
\hline & V & & $12 \sim 15$ & 13.5 & 3 & 10 & 208.33 & 104.17 & 0.50 & 156.25 & 229.17 & 1.10 & 0.50 & 0.69 \\
\hline & VI & & $15 \sim 18$ & 16.5 & 3 & 5 & 104.17 & 83.33 & 0.80 & 62.50 & 72.92 & 0.70 & 0.20 & 1.61 \\
\hline & VII & & $18 \sim 21$ & 19.5 & 3 & 1 & 20.83 & 20.83 & 1.00 & 10.42 & 10.42 & 0.50 & 0.00 & 1.00 \\
\hline
\end{tabular}

$x$ : 单位时间的龄级中值 (此处为径级中值 单位为 $\mathrm{cm}$ ) $\Delta_{\mathrm{x}}$ : 龄级宽度 (此处为径级宽度 单位为 $\mathrm{cm}$ 根据方枝柏种群的生物学特性，龄级 宽度划分标准以 $5 \mathrm{~cm}$ 为一龄级) $a_{x}$ :在 $x$ 龄级内现存的个体数 $l_{x}$ : 在 $\mathrm{x}$ 龄级开始时的标准化存活个体数 (以 1000 为基数 ), $l_{x}=a_{x} / a_{0} \times 1$ $000 d_{x}$ : 从 $x$ 到 $x+1$ 间隔期内标准化死亡数, $d_{x}=a_{x}-a_{x}+1 \quad q_{x}$ : 从 $x$ 到 $x+1$ 龄级间隔期间个体死亡率, $q_{x}=d_{x} / l_{x} \quad L_{x}$ : 从 $x$ 到 $x+1$ 龄级 间隔期间平均存活的个体数, $L_{x}=\left(l_{x}+l_{x}+1\right) / 2 \quad T_{x}: x$ 龄级及其以上各龄级存活的个体总数, $T_{x}=\Sigma L_{x} \quad e_{x}$ : 进入 $x$ 龄级个体的生命期望寿 命 , $e_{x}=T_{x} / l_{x} \quad S_{x}$ : 存活率, 即 $x+1$ 期存活个体数与 $x$ 期存活个体数之比, $S_{x}=l_{x}+1 / l_{x} \quad K_{x}$ : 致死力或消失率, 即从 $x$ 到 $x+1$ 期受到的阻力， $K_{x}=\ln \left(l_{x}\right)-\ln \left(l_{x}+1\right)$

$x$ : Midpoint age (here as midpoint $D B H, \mathrm{~cm}) \quad \triangle_{x}$ : Width of age (here as width of $\left.D B H, \mathrm{~cm}\right) \quad a_{x}$ : Number of survival individuals $\quad l_{x}$ : Proportion individuals surviving from the beginning to age $x, l_{x}=a_{x} / a_{0} \times 1000 \quad d_{x}$ : Number of dead individuals from age $x$ to $x+1, d_{x}=a_{x}-a_{x}+1 \quad q_{x}:$ Mortality rate from $x$ to $x+1, q_{x}=d_{x} / l_{x} \quad L_{x}$ : Mean number of survival individuals from age $x$ to $x+1, L_{x}=\left(l_{x}+l_{x}+1\right) / 2 \quad T_{x}$ : Total number of survival individuals from age $x, T_{x}=\Sigma L_{x} \quad e_{x}$ : Life expectancy at age $x, e_{x}=T_{x} / l_{x} \quad S_{x}$ : Age - specific survival, $S_{x}=l_{x}+1 / l_{x} \quad K_{x}:$ Age specific mortality,$K_{x}=\ln \left(l_{x}\right)-\ln \left(l_{x}+\right.$ 1)

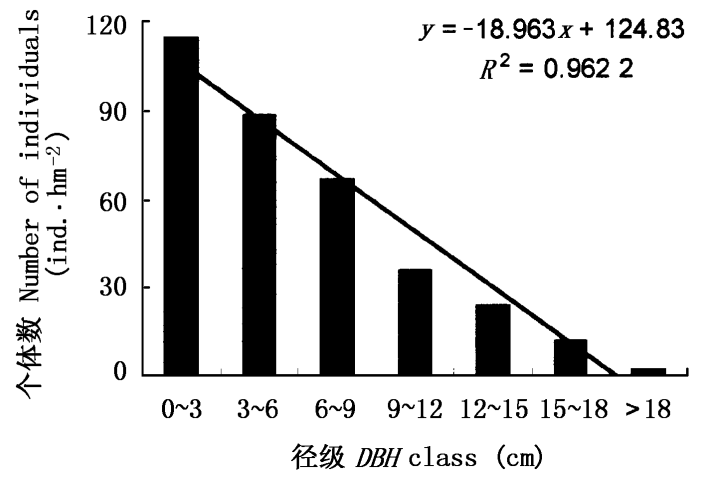

图 1 白马雪山阳坡方枝柏林线种群径级结构图

Fig. 1 Histogram of DBH classes of Sabina saltuaria population at timberline of south-facing slope in Baima Snow Mountain , Yunnan Province

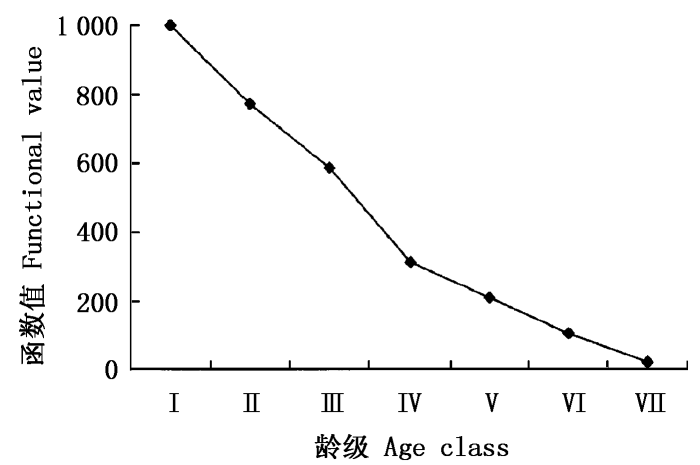

图 2 白马雪山阳坡林线方枝柏种群存活曲线

Fig.2 Survival curve of Sabina saltuaria population at timberline of south-facing slope in the Baima Snow Mountain , Yunnan Province
方枝柏种群的存活曲线介于 Deevey- II 型和 DeeveyIII型之间。如图 3 所示, 方枝柏的死亡率曲线和消 失率曲线变化趋势在龄级 I - VI间基本一致, 都在龄 级 III 出现一高峰; 从龄级 VI到龄级 VII 死亡率仍逐渐 上升，而消失率曲线则呈急剧下降趋势。整体而言， 死亡率和消失率都随着龄级的增加而呈现逐渐增加 的趋势。

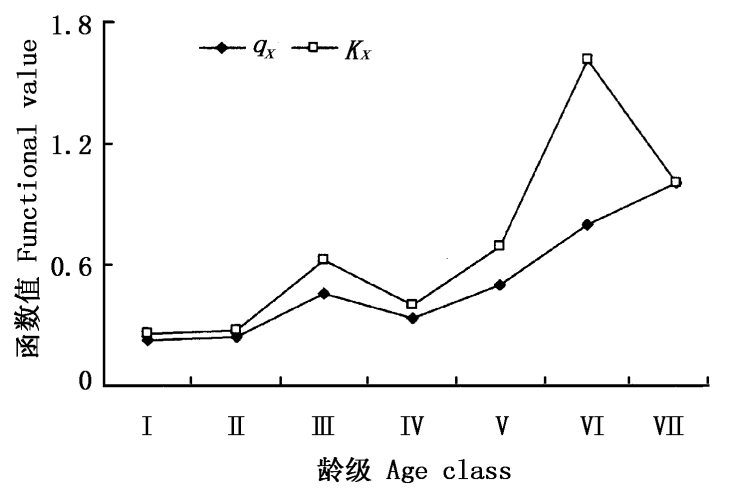

图 3 白马雪山阳坡林线方枝柏种群死亡率 $\left(q_{x}\right)$ 曲线及消失率 $\left(K_{x}\right)$ 曲线 Fig.3 Mortality rate $\left(q_{x}\right)$ and hazard rate $\left(K_{x}\right)$ of Sabina saltuaria population at timberline in the Baima Snow Mountain , Yunnan Province

\section{4 种群各龄级分布格局分析}

图 4 表示的是方枝柏种群在林线的 3 个龄级个 体在 $70 \mathrm{~m} \times 60 \mathrm{~m}$ 样地中的分布点图。由图 4 可见， 在林线的 3 个龄级中密度差异很明显, 其中幼苗 (图 4a)最少, 其密度仅为 17 株 $\mathrm{hm}^{-2}$,幼树 (图 4b) 为 107 株 $\mathrm{hm}^{-2}$, 成年树 (图 4c) 最多, 为 233 株 $\mathrm{hm}^{-2}$ ， 密度顺序是成年树 $>$ 幼树 > 幼苗。从图上看, 各龄 
级均具有一定程度的集群分布,但单从图 4 看不出 这种分布与尺度的关系。

60

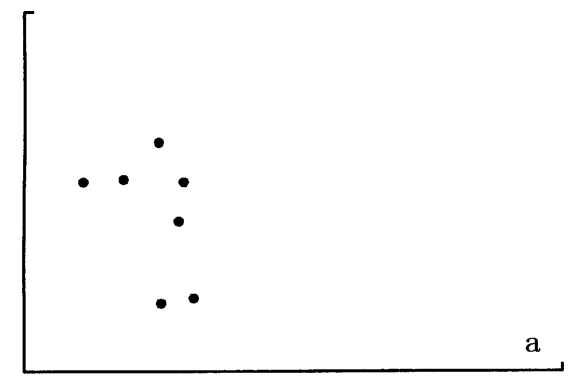

0

60

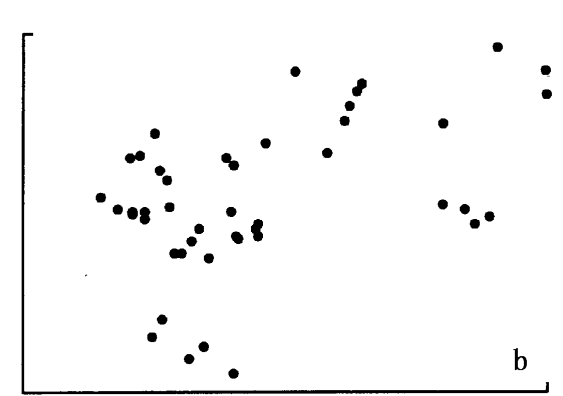

0

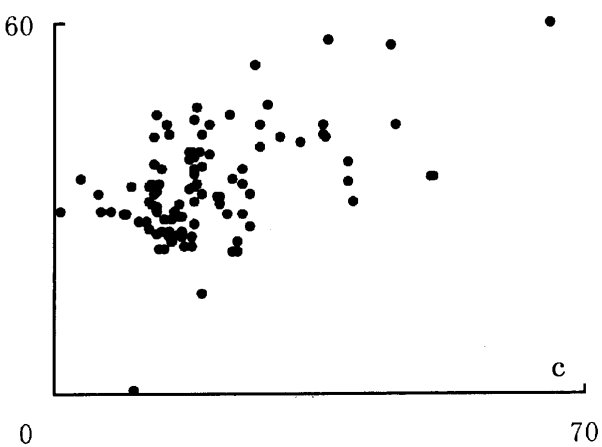

图 4 方枝柏各龄级个体在样方中的分布点图

Fig.4 Point pattern of individuals for different age classes of Sabina saltuaria

a. 幼苗 Seedlings b. 幼树 Saplings c. 成年树 Trees

方枝柏 3 个龄级的点格局分析结果见图 5。图 中实线为用实际数据计算的 $\hat{H}(t)$, 虚线为拟合的上 下包迹线, 包迹线即为置信水平, 当 $\hat{H}(t)$ 值大于上 包迹线值时, 说明个体显著偏离随机分布, 呈集群分 布; 当 $\hat{H}(t)$ 值小于下包迹线值时, 说明个体也显著 偏离随机分布, 但呈均匀分布。分析时林线各龄级 取 $t$ 的间隔为 $3 \mathrm{~m}, t$ 的最大值为样地最短边长的一 半即 $30 \mathrm{~m}$, 图的 $x$ 轴坐标 $t$ 用空间距离 $(\mathrm{m})$ 直接表 示。图 5 分别为林线幼苗、幼树和成年树的格局分 析结果。对于幼苗(图 5a)，在 $<14 \mathrm{~m}$ 的尺度内表现 为随机分布, 但在 $0 \sim 2 \mathrm{~m}$ 尺度内 $\hat{H}(t)$ 与下包迹线 重合, 随机分布不显著, 当尺度 $>14 \mathrm{~m}$ 时, 为聚集分
布; 对于幼树 (图 5b) 和成年树 (图 5c) 在所有尺度下 均呈显著的聚集分布，成年树的聚集强度显著大于 幼树。可见幼龄方枝柏在尺度小的情况下，其个体 呈随机分布, 但在大尺度情况下, 个体又呈集群分 布。随着方枝柏年龄的增长, 其空间分布显著地趋 于集群分布。

\section{5 各龄级间的关系分析}

方枝柏 3 个龄级间相互关系分析结果见图 6 , 图 6 的结构与图 5 相似，实线为实测的 $\hat{H}_{12}(t)$ 值，虚 线为拟合的上下包迹线。图 6a 为幼苗和幼树之间 的关系图，图 6b 为幼苗和成年树之间的关系图，图 $6 \mathrm{c}$ 为幼树和成年树之间的关系图。由图 6 可知，林 线区方枝柏各个龄级相互之间的关系相同, 在所有 尺度下都表现为显著的正相关，其中幼苗和成年树 的相关性最强, 幼苗和幼树相关性最弱。这也体现 林线区方枝柏种群不同年龄的个体之间虽然存在着 竞争，但对环境条件的要求具有一致性。

\section{3 结论与讨论}

白马雪山阳坡林线方枝柏种群幼苗数量非常 少, 仅 17 株 $\mathrm{hm}^{-2}$,分别为林线区幼树和成年树密 度的 $1 / 6$ 和 $1 / 14$ 。这可能与物种本身的生物学特 性、环境条件及人为干扰等因素相关。林线区方枝 柏幼苗主要来自土壤种子库, 球果种鳞苞鳞完全合 生 种子萌发有所滞后; 研究地坡度较大而且草本及 地被物覆盖度不及 $20 \%$ 球果可能在重力作用下沿 山坡下移, 而在降雪或降水的影响下，一部分种子可 能随地表水土流失损失掉; 林线区的低温、强辐射、 冻害等对幼苗尤其是当年生幼苗也会造成很大伤害 (Matthew et al. 2002)。另外，林线区人类活动对幼 苗命运也起到了一定的决定作用。阳坡森林郁闭度 低于阴坡, 灌丛数量和种类较阴坡少, 融雪早且积雪 时间短，所以阳坡成为当地牧民的夏季牧场，牲畜的 践踏、啃食也使得幼苗数量大大减少。我们不得不 承认这是一个不容乐观的信号, 高山林线区森林一 旦破坏就很难恢复, 尤其阳坡林线, 柏类生长非常缓 慢，在短时间内很难有效更新和恢复，因此适度控制 人为干扰以减少其直接和间接破坏对于高山生态系 统的生态恢复意义重大。

由于生命表的编制基于径级数据，其中 $0 \sim 3 \mathrm{~cm}$ 的第 I 龄级包括了幼苗和一部分幼树, 所以第 I 和 第 II 龄级都显示出较高的生命期望值, 表明幼苗在 经历严酷的环境篮及人为干扰后一旦长成幼树, 其 生存能力便极大提高。生存分析表明存活曲线介于 

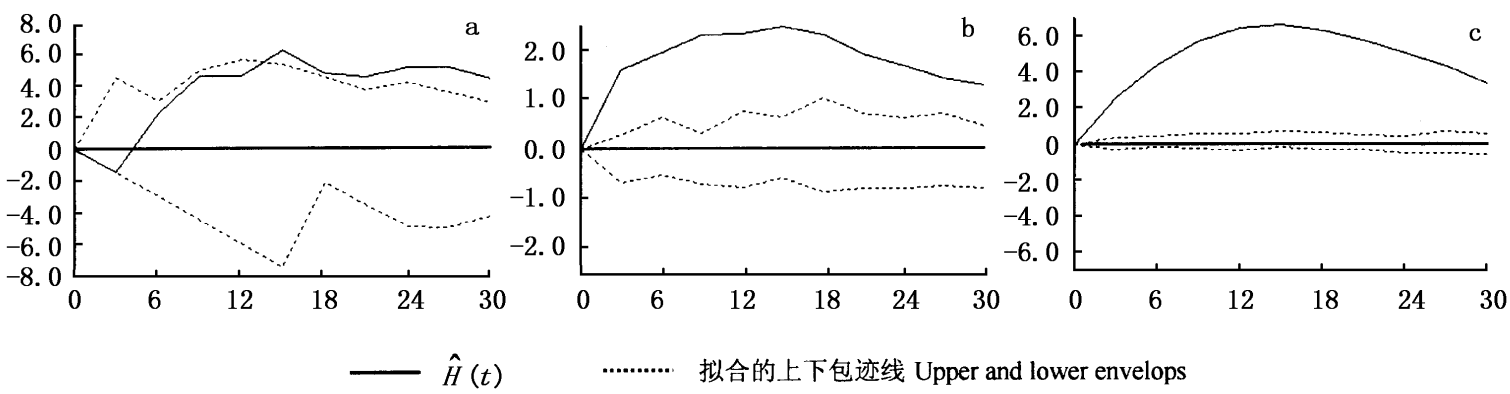

拟合的上下包迹线 Upper and lower envelops

图 5 方枝柏各龄级点格局分析结果图

Fig.5 Point pattern analysis for three age classes of Sabina saltuaria at timberline of Baima Snow Mountain a、b、c : 见图 4 See Fig. 4
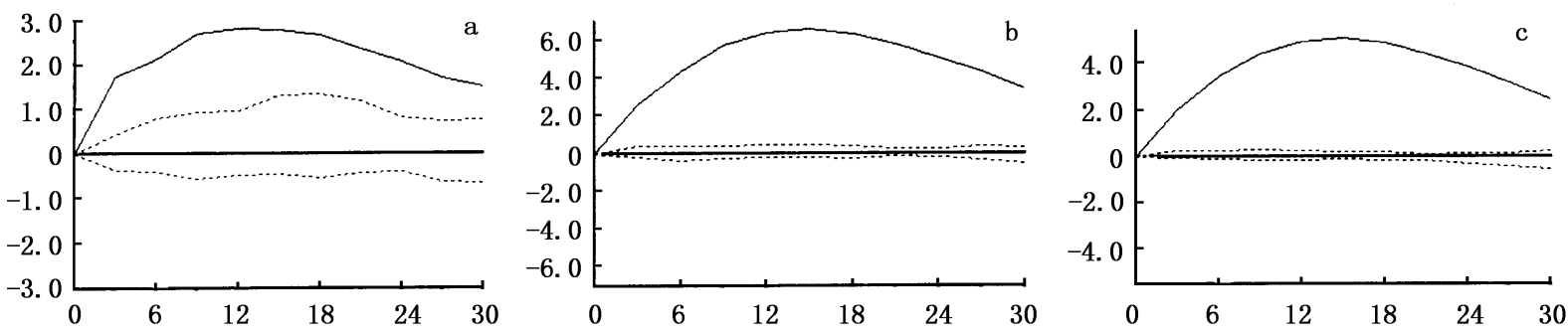

$-\hat{H}_{12}(t)$

拟合的上下包迹线 Upper and lower envelops

图 6 方枝柏各龄级间关系的点格局分析

Fig.6 Point pattern analysis of paired age-class associations of Sabina saltuaria at timberline of Baima Snow Mountain a、b、c : 见图 4 See Fig. 4

Deevey- II 型和 Deevey- III 型之间，种群结构接近稳定 型。死亡率曲线和消失率曲线都在龄级 III 出现一个 高峰, 可能由于方枝柏处于青壮年期, 个体对营养的 需求不断增大 对空间、光照和养分等生存因子空前 激烈的竞争导致了强烈的自疏过程，死亡率有所上 升 此后, 进入冠层的个体生存条件明显改善, 死亡 率略有降低。但由于林线附近气候严酷，如强光照、 低温和水份胁迫等, 加上干扰等引起的机械性损伤 很难被修复 植物体内糖和氨基酸的合成过程不能 达到组织更新所需的最小速率 (Benecke \& Havranek , 1980 ;James et al . 1994) ,所以各个龄级仍保持一定 的死亡率, 种群逐渐削弱, 本研究中, 只有极个别个 体能存活到第 VII龄级。方枝柏生长缓慢且生长环境 恶劣, 因此为防止阳坡林线下降,应加强保护。

在白马雪山阳坡林线区, 方枝柏种群各龄级的 空间格局基本上是聚集型; 各龄级关系密切, 都呈显 著正相关, 其中幼苗和成年树的相关性最强 幼苗和 幼树相关性最弱。树种在不同发育阶段、不同年龄
级会表现不同的空间格局, 这与森林群落的自然稀 疏过程、干扰格局以及环境的变化有密切关系 (Greig-Smith, 1983 张金屯和孟东平,2004)。在研究 区, 方枝柏种群未曾受到火灾及病虫害等干扰, 因此 各龄级格局差异不显著。但由于种群生物学特性、 环境条件及人为干扰等的综合影响, 种群随龄级增 加聚集尺度和聚集强度都增加。由上述分析可知， 在多种因素综合作用下，林线区方枝柏幼苗数量非 常少, 因此小尺度下呈随机分布, 在大尺度下趋于随 机分布。成年树适应力最强, 数量最多, 表现出了最 大的聚集强度和尺度。在林线区恶劣的自然条件下 幼苗的生长依赖于成年树为其创造的林下生境 (Matthew et al. 2002), 以减少强光、霜冻等不利因 素的威胁, 由此也不难理解幼苗与成年树间显示出 了最为密切的相关性。而幼苗一旦经历环境的选择 长成幼树, 其适应能力相对增强, 与成年树的关系也 逐渐由依赖转为竞争，且由于自疏的影响，二者的关 联性有所下降。另外, 各龄级间显著的正相关也表 
明不同龄级个体在空间交错分布, 这样有利于对各 种资源的充分利用(张金屯和孟东平，2004），对整个 种群的生存和发展是有利的, 这也反映了高山生态 系统恶劣生境中种群的一种适应对策。

本研究采用森林罗盘仪与测距仪相结合的方法 对林线区方枝柏种群进行每木定位, 测距仪利用超 声波信号来测定距离, 可精确到 $0.01 \mathrm{~m}$, 这样既避 免了由于林线区高覆盖率的灌木对相邻格子布置的 影响而降低结果的准确率, 又极大地降低了工作强 度, 不失为高海拔区恶劣条件下开展类似工作的推 荐之法。本研究也表明空间点格局分析方法 (Ripley's K 系数法) 能够很好地体现种群不同尺度下的 分布格局和格局强度, 并能定量描述龄级间的相关 性, 是种群格局分析的有效方法。

\section{参 考 文 献}

Benecke U, Havranek WM (1980). Gas-exchange of trees at altitudes up to timberline. In: Benecke U, Davis MR eds. Mountain Environments and Subalpine Tree Growth. New Zealand Forest Research Institute Technical Paper, New Zealand, 70, 195 212.

Chen WN (陈文年), Wu N (吴宁), Luo P (罗鹏) (2003). Species diversity and arbor population distribution pattern of Sabina przewalskii community in the forest-grassland ecotone in the watershed of upper Minjiang River. Chinese Journal of Applied and Environmental Biology (应用与环境生物学报), 9, 221 - 225. (in Chinese with English abstract)

Cheng W (程伟), Luo P (罗鹏), Wu N (吴宁) (2005a) . Ecological characteristics of Minjiang fir (Abies faxoniana) population near timberline on upper Minjiang River. Chinese Journal of Applied and Environmental Biology (应用与环境生物学报), 11, 300 - 303. (in Chinese with English abstract)

Cheng W (程伟), Wu N (吴宁), Luo P (罗鹏) (2005b). Survival analysis of Abies faxoniana population near timberline on the upper Minjiang River. Acta Phytoecologica Sinica (植物生态学 报), 29, 349 - 353. (in Chinese with English abstract)

Condit R, Ashton PS, Baker P, Bunyavejchewin S, Gunatilleke S, Gunatilleke N, Hubbell SP, Foster RB, Itoh A, LaFrankie JV, Lee HS, Losos E, Manokaran N, Sukumar R, Yamakura T $(2000)$. Spatial patterns in the distribution of tropical tree species. Science, 288, $1414-1418$.

Dale MRT (1999). Spatial Patterns Analysis in Plant Ecology. Cambridge University Press, Cambridge.

Editorial Committee of Vegetation of China (中国植被编辑委员 会) (1980). Vegetation of China (中国植被). Science Press,
Beijing, 209 - 210. (in Chinese)

Editorial Committee of Vegetation of Sichuan (四川植被协作组) (1980). Vegetation of Sichuan (四川植被). Sichuan People's Press, Chengdu. (in Chinese)

Forestry Department of Yunnan Province（云南省林业厅） (2003). Baima Snow Mountain National Nature Reserve (白马 雪山国家级自然保护区). Yunnan Minority Press, Kunming,

7. (in Chinese)

Frost I, Rydin H (2000). Spatial pattern and size distribution of the animal-dispersed Quercus rubur in two spruce-dominated forests. Ecoscience, 7, $38-44$.

Getis A, Franklin J (1987). Second-order neighborhood analysis of mapped point patterns. Ecology, 68, 473-477.

Greig-Smith P (1983). Quantitative Plant Ecology. Blackwell, London, $21-36$.

James JC, Grace J, Hoad SP (1994). Growth and photosynthesis of Pinus sylvestris at its altitudinal in Scotland. Journal of Ecology, $82,297-306$.

Jiang H (江洪) (1992). Population Ecology of Spruce (云杉种群 生态学). China Forestry Publishing House, Beijing, 8-32. (in Chinese)

Körner C (1998) . A re-assessment of high elevation treeline positions and their explanation. Oecologia, 115, $445-459$.

Körner C (1999). Alpine Plant Life-Functional Plant of Ecology of High Mountain Ecosystem. Springer-Verlag, Berlin, Heidelberg.

Lloyd AH, Graumlich LJ (1997). Holocene dynamics of treeline forests in the Sierra Nevada. Ecology, 78, $1199-1210$.

Matthew JG, William SK, Catherine RA (2002) . Conifer seedling distribution and survival in an alpine- treeline ecotone. Plant Ecology, 162, 157 - 168 .

Shen ZH (沈泽昊), Fang JY (方精云), Liu ZL (刘增力), Wu J (伍杰) (2001). Structure and dynamics of Abies fabri population near the alpine timberline in Hailuo Clough of Gongga Mountain. Acta Botanica Sinica (植物学报), 43, 1288-1293. (in Chinese with English abstract)

Tranquillini W (1979). Physiological Ecology of Alpine Timberline. Springer-Verlag, Berlin, $1-2$.

Wu N (吴宁), Liu ZG (刘照光) (1998). Probing into the causes of geographical pattern of subalpine vegetation on the eastern Qinghai-Tibetan Plateau. Chinese Journal of Applied and Environmental Biology (应用与环境生物学报)，4, 290 - 297.（in Chinese with English abstract)

$\mathrm{Xu}$ XH (徐学红), Yu MJ (于明坚), Hu ZH (胡正华), Li MH (李铭红), Zhang FG (张方钢) (2005). The structure and dynamics of Castanopsis eyrei population in Gutian Mountain Natural 
Reserve in Zhejiang, East China. Acta Ecologica Sinica (生态 学报), 25,645-653. (in Chinese with English abstract) Yan GQ (问桂琴), Zhao GF (赵桂仿), Hu ZH (胡正海), Yue M (岳明) (2001). Population structure and dynamics of Larix chinensis in Qinling Mountain. Chinese Journal of Applied Ecology (应用生态学报), 12,824-828. (in Chinese with English abstract)

Zhang JT (张金屯) (1998). Analysis of spatial point pattern for plant species. Acta Phytoecologica Sinica (植物生态学报), 22, $344-349$. (in Chinese with English abstract)
Zhang JT (张金屯), Meng DP (孟东平) (2004). Spatial pattern analysis of individuals in different age-class of Larix principis-rupprechtii in Luya Mountain Reserve, Shanxi, China. Acta Ecologica Sinica (生态学报), 24, 35-40. (in Chinese with English abstract)

Zhao CM (赵常明), Chen QH (陈庆恒), Qiao YK (乔永康), Pan KW (潘开文) (2004). Structure and spatial pattern of a natural Abies faxoniana population on the eastern edge of Qinghai-Tibetan Plateau. Acta Phytoecologica Sinica (植物生态学 报), 28, $341-350$. (in Chinese with English abstract) 